\title{
Positive and negative cue redundancy in multiple cue probability learning
}

\author{
NEAL SCHMITT* \\ Michigan State University, East Lansing, Michigan 48823 \\ and \\ ARTHUR L. DUDYCHA \\ Purdue University, West Lafayette, Indiana
}

\begin{abstract}
In two separate experiments, 80 introductory psychology students completed a two-cue probability learning task in which cue intercorrelation was positive or negative and cue validities were positive or negative. Results indicated that $\mathrm{Ss}$ did not learn the negative validity tasks. In addition, there were no significant differences between groups in achievement due to cue intercorrelation within any one experiment. However, the positive redundancy groups were significantly more consistent in the employment of their decisian strategy than the negative redundancy groups when cue validities were positive. The negative redundancy groups were superior to positive redundancy groups in matching; again, when cue validities were positive.
\end{abstract}

A puzzling problem confronting researchers in the area of multiple cue probability learning (MCPL) has been the effect of cue intercorrelation or redundancy of information. Many of the early studies utilizing the lens model paradigm avoided the problem by utilizing only orthogonal cues in their studies. More recently, the investigators interested in increasing the "real world relevance" of their experimental manipulations have recognized the necessity of investigating the effect of the cue redundancy factor on MCPL (Naylor \& Schenck, 1968; Schenck, 1968; Armelius \& Lenntoft, 1970; and Knowles, Hammond, Stewart, \& Summers, 1971).

The results of all three of these studies have been somewhat misleading and ambiguous. An examination of the design characteristics for these studies reveals that the cue-redundancy manipulation was confounded to a greater or a lesser extent with the cue validities and/or task predictability, as well as with the cue-regression weights. For example, Schenck (1968) required his Ss to learn to predict a criterion on the basis of two equally valid orthogonal cues whose multiple correlation with the criterion was .90 or .70 . After 120 trials, or after learning had taken place, cue intercorrelation was shifted from .00 to .40 or .80 . In order to maintain the initial level of total task predictability $(.90$ or .70$)$ cue validites were necessarily increased, thus confounding the effect of cue intercorrelation with cue validity. Schenck found, under these conditions, that increasing the intercorrelation increased the consistency and achievement of Ss.

Subsequent investigators (Armelius \& Lenntoft, 1970)

*Requests for reprints should be made to Neal Schmitt, Department of Psychology, Olds Hall, Michigan State University, East Lansing, Michigan $\mathbf{4 8 8 2 4 .}$ also failed to eliminate or circumvent the confounding problem experienced by Schenck (1968). Knowles et al (1971) added a new dimension to the study of cue redundancy by investigating the effect of both positive and negative cue intercorrelation. Although their research design maintained the task predictability $\left(\mathrm{R}_{\mathrm{e}}^{2}=.84\right)$ and the proportionality of the regression weights $\left(2 b_{1}=b_{2}\right)$ across conditions, unfortunately the cue validities were quite disparate. Analyzing only the dependent variable of achievement (see L. Dudycha \& Naylor, 1966, for an explanation of the lens model performance indices), Knowles et al found slower learning in the negative redundancy conditions, but at the end of 50 trials, the mean level of achievement for all groups was quite similar. Also performance in the low negative redundancy group $\left(\mathrm{r}_{12}=-.40\right)$ was superior to performance in the high negative redundancy group $\left(\mathrm{r}_{12}=-80\right)$. They concluded that negative intercorrelation between cues does not facilitate learning, but may actually impede learning.

Recently, Dudycha, Dudycha, and Schmitt (1974) have shown that this confounding of total task predictability with cue validities is not necessary and that the relationship between task predictability and cue intercorrelation is curvilinear yielding two separate levels of cue intercorrelation for any pair of unequal cue-criterion validities. The purpose of the present study was to assess the effect of positive and negative cue intercorrelation in MCPL when cue validities and task predictability are held constant.

\section{METHOD}

Two separate 2 (levels of cue intercorrelation) by 2 (positive or negative cue validities) experiments were conducted. Within 
each experiment, total task predictability $\left(\mathrm{R}_{\mathrm{e}}^{2}\right)$ and the cue validities $\left(\mathrm{r}_{\mathrm{e} 1}\right.$ and $\left.\mathrm{r}_{\mathrm{e} 2}\right)$ were held constant. Experiment $I$ represented a highly predictable system $\left(\mathrm{R}_{\mathrm{e}}^{2}=90\right)$, while in Experiment II, $\mathrm{R}_{\mathrm{e}}^{2}$ was only .70. Table 1 gives the task characteristics for each of the eight conditions.

Ten Ss per condition predicted a numerical criterion on the basis of two numerical cues for a total of 150 trials with outcome feedback after each trial. In any one trial, cues were presented on a screen for approximately $15 \mathrm{sec}$, during which time Ss were required to estimate the "criterion." The "correct" answer along with the cues was then displayed for approximately $10 \mathrm{sec}$. Cue and criterion values were generated by computer and exhibited the desired correlative values within \pm .05 for any one group of 25 trials. All three variables had means of 50 and standard deviations of 10.

The three dependent variables-subject achievement $\left(r_{\mathrm{a}}\right)$, consistency $\left(r_{s}\right)$, and matching $\left(r_{m}\right)$ were computed for each 25 -trial block and transformed to Fisher $Z$ values. Subject achievement represents the correlation of the $S$ 's responses with the criterion. Subject consistency is the correlation between the S's responses and his predicted responses computed utilizing the $S$ 's regression equation; therefore it represents how consistently the $S$ utilizes a strategy he has developed over a block of trials. Subject matching is obtained by correlating the S's predicted responses with the best criterion estimates (as defined by the multiple regression equation relating the cues to the criterion). Tucker (1964) and Schenck and Naylor (1965) have shown that $\mathrm{S}$ achievement is a product of the S's consistency, his matching, and the predictability afforded by the task when the residual variance of the criterion system is generated randomly thus eliminating nonlinear covariance.

A 2 by 2 by 6 (two levels of intercorrelation-I, two levels of the sign of the cue validities $-S$, and six trial blocks $-B$ ) repeated measures analysis of variance was performed on each of these indices. In all analyses, all factors were considered fixed.

\section{RESULTS}

Results of the analyses of variance performed on each of the dependent measures (achievement, consistency, and matching) are summarized for each experiment and then the results of the two experiments are compared.

Experiment $\mathrm{J}: \mathrm{r}_{\mathrm{e} 1}= \pm .60, \mathrm{r}_{\mathrm{e} 2}= \pm .20, \mathrm{r}_{12}=.89$ or $-.63, \mathrm{R}_{\mathrm{e}}^{2}=90$

Results of the analyses of variance for Experiment $I$ are presented in Table 2. Significant main effects due
Table 1

Cue Validities, Cue Intercorrelations, and Total Task Predictability for All Eight Conditions*

\begin{tabular}{crrrrrr}
\hline Condition & $\mathrm{r}_{\mathrm{e} 1}$ & $\mathrm{I}_{\mathrm{e} 2}$ & $\mathrm{r}_{12}$ & $\mathrm{~b}_{\mathrm{e} 1}$ & $\mathrm{~b}_{\mathrm{e} 2}$ & $\mathrm{R}_{\mathrm{e}}^{2}$ \\
\hline \multicolumn{7}{c}{ Experiment $\mathrm{I}$} \\
1 & .60 & .20 & .89 & 2.030 & -1.606 & .90 \\
2 & -.60 & -.20 & .89 & -2.030 & 1.606 & .90 \\
3 & .60 & .20 & -.63 & 1.204 & .958 & .90 \\
4 & -.60 & -.20 & -.63 & -1.204 & -.958 & .90 \\
& \multicolumn{5}{c}{ Experiment II } \\
5 & .60 & .20 & .85 & 1.549 & -1.117 & .70 \\
6 & -.60 & -.20 & .85 & -1.549 & 1.117 & .70 \\
7 & .60 & .20 & -.51 & .949 & .680 & .70 \\
8 & -.60 & -.20 & -.51 & .949 & -.680 & .70 \\
\hline
\end{tabular}

*Following the notation of Dudycha and Naylor (1966), $r_{e 1}$ and $r_{e 2}$ equal the correlation of Cues 1 and 2 , respectively, with the criterion; $r_{12}$ equals the cue intercorrelation; $b_{e 1}$ and $b_{e 2}$ represent the beta weights of Cues 1 and 2 obtained by computing the regression equation relating the cues to the criterion; and $R_{e}^{2}$ represents the multiple correlation between the cues and the criterion or total task predictability.

to the sign of the cue validities and trial block as well as a significant interaction between these two variables were observed for Ss' achievement. A test for simple main effects within the $S$ by $B$ interaction revealed that Ss significantly improve their estimates of the criterion across trial blocks when the cue validities were positive $[F(5,180)=4.48, p<.01]$, but not when the cue validities were negative. The sign of the cue validities was significant at all blocks. Ss' achievement was not significantly different between the positive and negative redundancy conditions for either positive or negative cue validities (see Fig. 1)

For the consistency index, all three main effects were significant. In addition, the $I$ by $S$ and the $S$ by $B$ interactions were significant. Tests for simple main effects within the I by S interaction indicated that only intercorrelation of cues was a significant factor when cue validities were positive $[F(1,36)=30.46, p<.01]$ (see Fig. 2). The blocks factor within the $S$ by $B$ interaction

Table 2

Results of Analysis of Variance on Subject Achievement, Consistency, and Matching: Experiment I

\begin{tabular}{|c|c|c|c|c|c|c|c|}
\hline \multirow[b]{2}{*}{ Source } & \multirow[b]{2}{*}{$\mathrm{df}$} & \multicolumn{2}{|c|}{ Achievement } & \multicolumn{2}{|c|}{ Consistency } & \multicolumn{2}{|c|}{ Matching } \\
\hline & & MS & $\mathrm{F}$ & MS & $\mathrm{F}$ & MS & $\mathrm{F}$ \\
\hline Between Ss & 39 & .114 & & .845 & & 3.527 & \\
\hline I & 1 & .002 & .017 & 10.012 & $27.867 * *$ & 5.927 & $5.448^{*}$ \\
\hline $\mathrm{S}$ & 1 & 10.108 & $73.001 * *$ & 7.739 & $21.539 * *$ & 78.860 & $72.482 * *$ \\
\hline IS & 1 & .003 & .019 & 2.287 & $6.365^{*}$ & 13.589 & $12.490^{* *}$ \\
\hline Error & 36 & .138 & & .359 & & 1.088 & \\
\hline Within Ss & 200 & .061 & & .099 & & .817 & \\
\hline B & 5 & .127 & $2.292^{*}$ & .379 & $4.341 * *$ & .740 & .895 \\
\hline IB & 5 & .076 & 1.378 & .057 & .648 & 1.043 & 1.261 \\
\hline SB & 5 & .158 & $2.856^{*}$ & .238 & $2.727^{*}$ & .849 & 1.026 \\
\hline ISB & 5 & .098 & 1.769 & .150 & 1.718 & .278 & .336 \\
\hline Error & 180 & .055 & & .087 & & .827 & \\
\hline
\end{tabular}




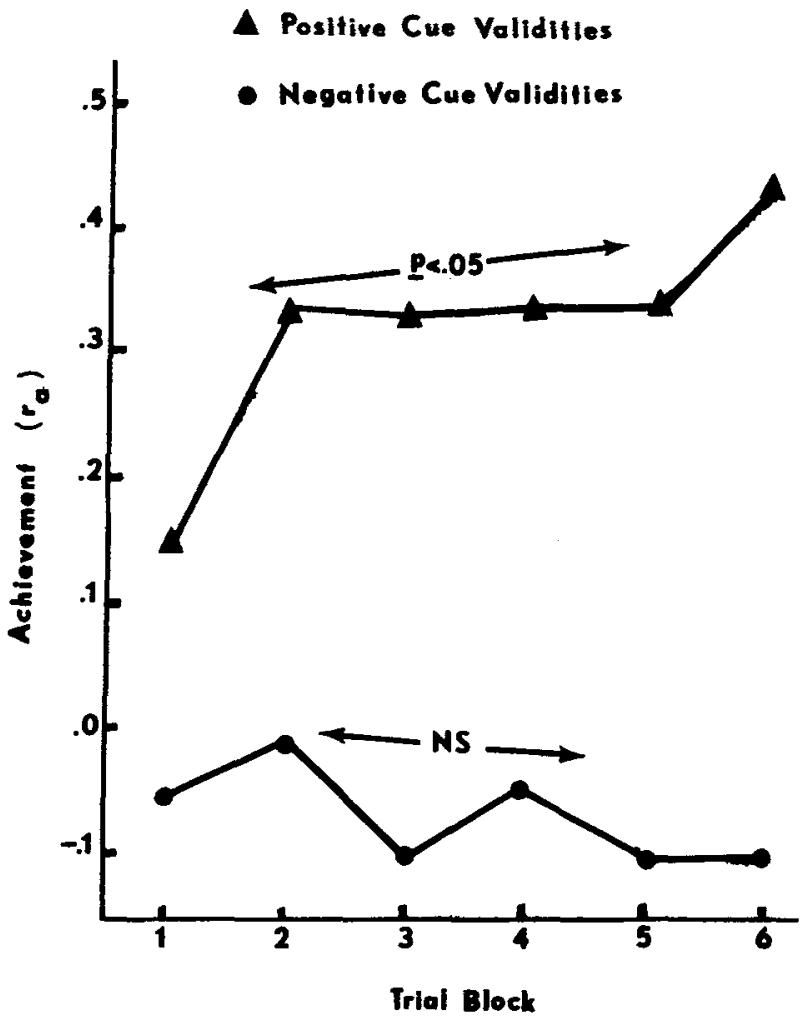

Fig. 1. S achievement as a function of the cue validites and trial block-Experiment 1 .

was also significant only when the cue validities were positive $[F(5,180)=6.71, p<.01]$ (see Fig. 3). The effect of the sign of the cue validities was significant at all blocks except the first $(p<.01)$. After the first block of trials, the positive validity and positive intercorrelation groups were superior in consistency to the negative validity and negative intercorrelation groups.

The analysis of variance on the matching index indicated that $S s$ in the negative intercorrelation conditions were significantly more capable of matching than those in the positive intercorrelation groups. There was also a significant main effect due to the sign of the cue validities as well as a significant $I$ by $S$ interaction. Tests for simple main effects within the I by $S$ interaction revealed that Ss' performance in the negative intercorrelation groups was significantly greater than that of positive intercorrelation groups only when the cue validities were positive $[F(1,36)=17.24, p<.01]$ (see Fig. 4).

Experiment II: $\mathrm{r}_{\mathrm{e} 1}= \pm .60, \mathrm{r}_{\mathrm{e} 2}= \pm .20, \mathrm{r}_{12}=.85$ or $-.51, \mathrm{R}_{\mathrm{e}}^{2}=.70$

For Ss' achievement in Experiment II, only the main effect due to the sign of the cue validities was significant (see Table 3).

When considering Ss' consistency, the results are similar to those of Experiment I. Ss in the negative



Fig. 2. S consistency as a function of the sign of the cue validities and cue intercorrelation-Experiment 1.

redundancy conditions were much less consistent than those in the positive redundancy conditions when cue validities were positive. In analyzing the significant I by $S$ interaction (see Fig. 5), it was found that the simple main effect of intercorrelation for positive cue validities was significant $[F(1,36)=17.60, p<.01]$. Again, positive validity and positive intercorrelation groups were superior to negative validity and negative intercorrelation groups.

Analysis of Ss' matching also revealed results similar to Experiment I. The main effect due to the sign of the



Fig. 3. S consistency as a function of the sign of the cue validities and cue intercorrelation-Experiment 1. 




Fig. 4. $S$ matching as a function of the sign of the cue validities and cue intercorrelation-Experiment 1.

cue validities was significant as well as the interaction of cue intercorrelation and the sign of the cue validities. As in Experiment $I$, the simple main effect due to intercorrelation for positive validity groups was significant $[F(1,36)=10.60, p<.01]$, because of the superior matching of $S s$ in negative intercorrelation groups (see Fig. 6).

\section{Results Across Experiments}

The results of the analyses within each of the experiments are remarkably consistent. Analyses on all



Fig. 5. $S$ consistency as a function of the sign of the cue validities and cue intercorrelation-Experiment 2.

three dependent variables are interesting in that though there are no differences for achievement due to intercorrelation, the negative redundancy groups are less consistent, but better at matching than the positive redundancy group; while the positive redundancy groups are highly consistent, but poor at matching. This is clearly seen in Figs. 7 and 8, which are summaries of Ss' performance on all three indices for all six trial blocks for both experiments.

Even though the total task predictability in the two experiments was different $\left(\mathrm{R}_{\mathrm{e}}^{2}=.90\right.$ vs .70$)$, it is also interesting that $\mathrm{Ss}^{\prime}$ achievement, consistency, and matching reached nearly the same levels in both groups indicating that $S$ sere not utilizing all the extra predictability afforded by the ecological properties of the task in Experiment I. Ss' achievement improved significantly over blocks for the high predictabilitv groups $\left(R_{e}^{2}=.90\right)$ only, but not nearly as much as would

Table 3

Results of Analyses of Variance on Subject Achievement, Consistency, and Matching: Experiment II

\begin{tabular}{|c|c|c|c|c|c|c|c|}
\hline \multirow[b]{2}{*}{ Source } & \multirow[b]{2}{*}{$\mathrm{df}$} & \multicolumn{2}{|c|}{ Achievement } & \multicolumn{2}{|c|}{ Consistency } & \multicolumn{2}{|c|}{ Matching } \\
\hline & & MS & $\mathrm{F}$ & MS & $\mathrm{F}$ & MS & $\mathrm{F}$ \\
\hline Between Ss & 39 & .271 & & .477 & & 4.304 & \\
\hline I & 1 & .022 & .215 & 2.446 & $10.971^{* *}$ & 2.685 & 1.533 \\
\hline$S$ & 1 & 6.706 & $66.704^{* *}$ & 6.587 & $29.541 * *$ & 82.273 & $46.969^{* *}$ \\
\hline IS & 1 & .237 & 2.355 & 1.531 & $6.868 * *$ & 19.853 & $11.334 * *$ \\
\hline Error & 36 & .101 & & .223 & & 1.752 & \\
\hline Within Ss & 200 & .051 & & .058 & & .982 & \\
\hline $\mathrm{B}$ & 5 & .071 & 1.441 & .102 & 1.781 & .954 & .968 \\
\hline IB & 5 & .040 & .815 & .063 & 1.088 & .621 & .631 \\
\hline SB & 5 & .090 & 1.849 & .067 & 1.165 & 1.559 & 1.582 \\
\hline ISB & 5 & .091 & 1.851 & .019 & .328 & .686 & .696 \\
\hline Error & 180 & .049 & & .058 & & .985 & \\
\hline
\end{tabular}




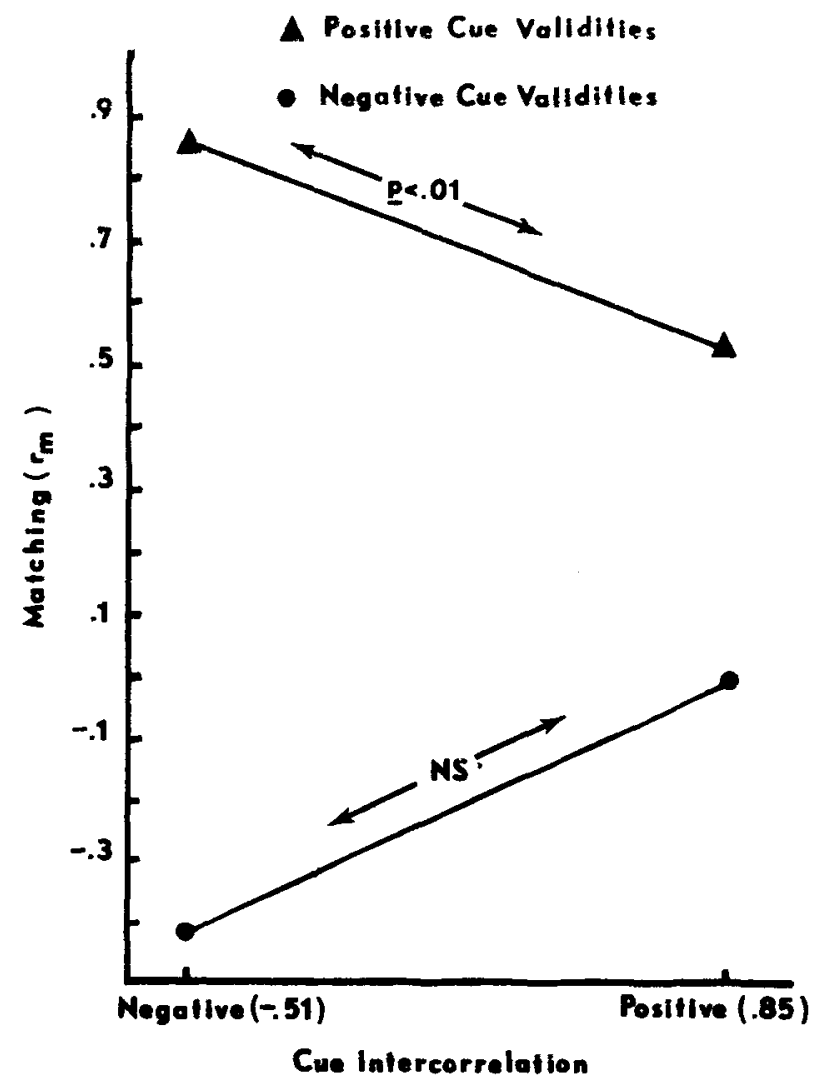

Fig. 6. $S$ matching as a function of the sign of the cue validities and cue intercorrelation-Experiment 2. be predicted by the statistical properties of the task.

A final point of interest is the manner in which the Ss utitize cues in the various conditions. Since the cues have different validities, they also have different regression weights and the differences in regression weights increase with increasing positive intercorrelation. As can be seen in $\mathrm{Fig} .9$, the differences in average weight, given to the cues by the Ss under the various levels of intercorrelation, do not match the differences in the task. This is expressed in a decrease in matching in the high positive redundancy groups. It can also be seen that Ss' beta weights more closely approximate cue-criterion correlations, though Ss do not appear to be discriminating between more and less valid cues. Ss' inability to match in high redundancy conditions is compensated for by their more consistent behavior in these conditions.

\section{DISCUSSION}

The fact that $\mathrm{Ss}^{\prime}$ achievement was attained in different ways in negative and positive redundancy groups is interpreted as evidence that $S$ s are not attending to cue beta weights and that cue-criterion correlations may be more important. The lack of confounding of cue intercorrelation with cue validities in this research, and the results of the present study, certainly extend our understanding of the effect of redundancy (positive or negative) on learning.

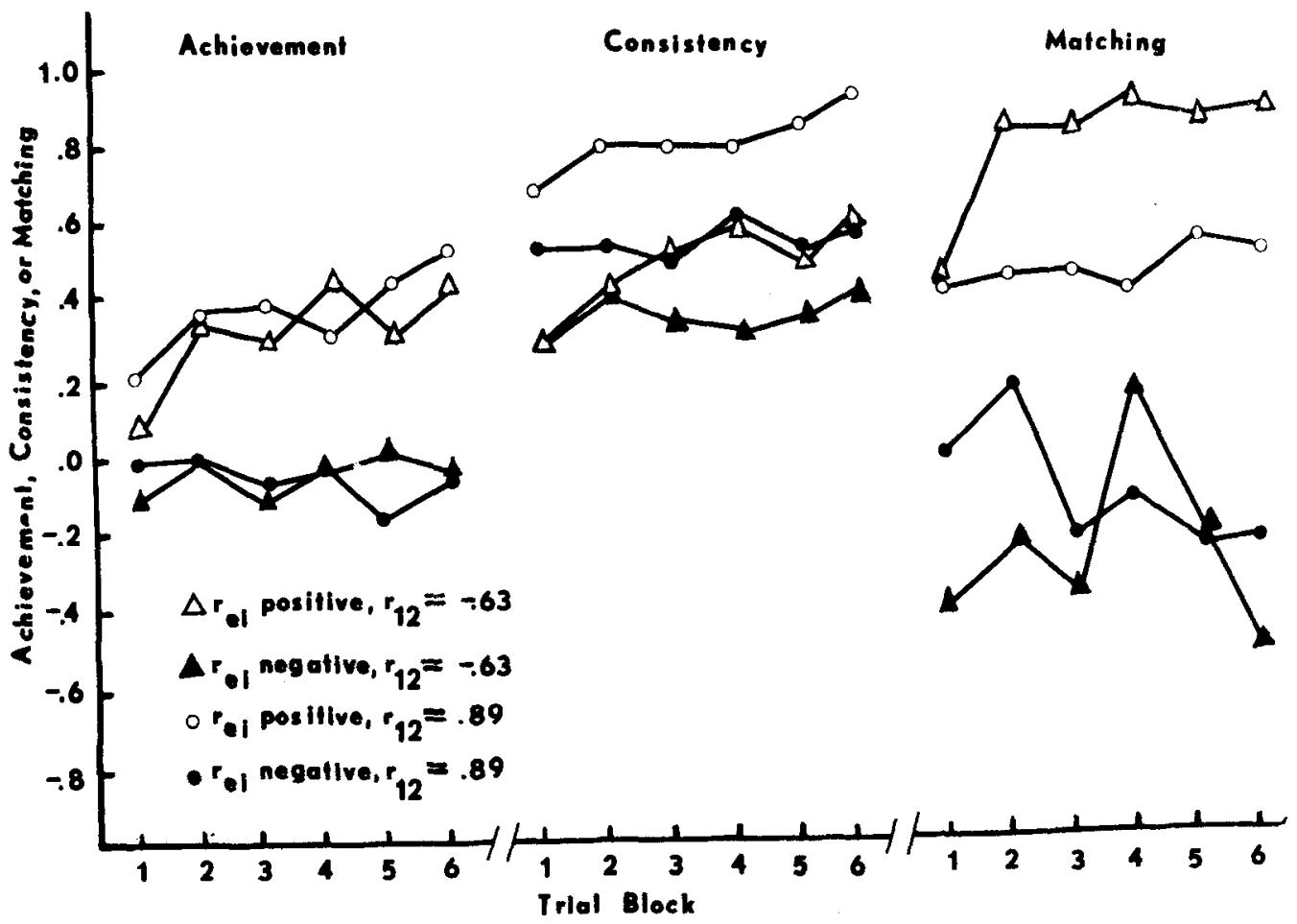

Fig. 7. S achievement, consistency, and matching as a function of the sign of the cue validities, cue intercorrelation, and trial block-Experiment 1 . 


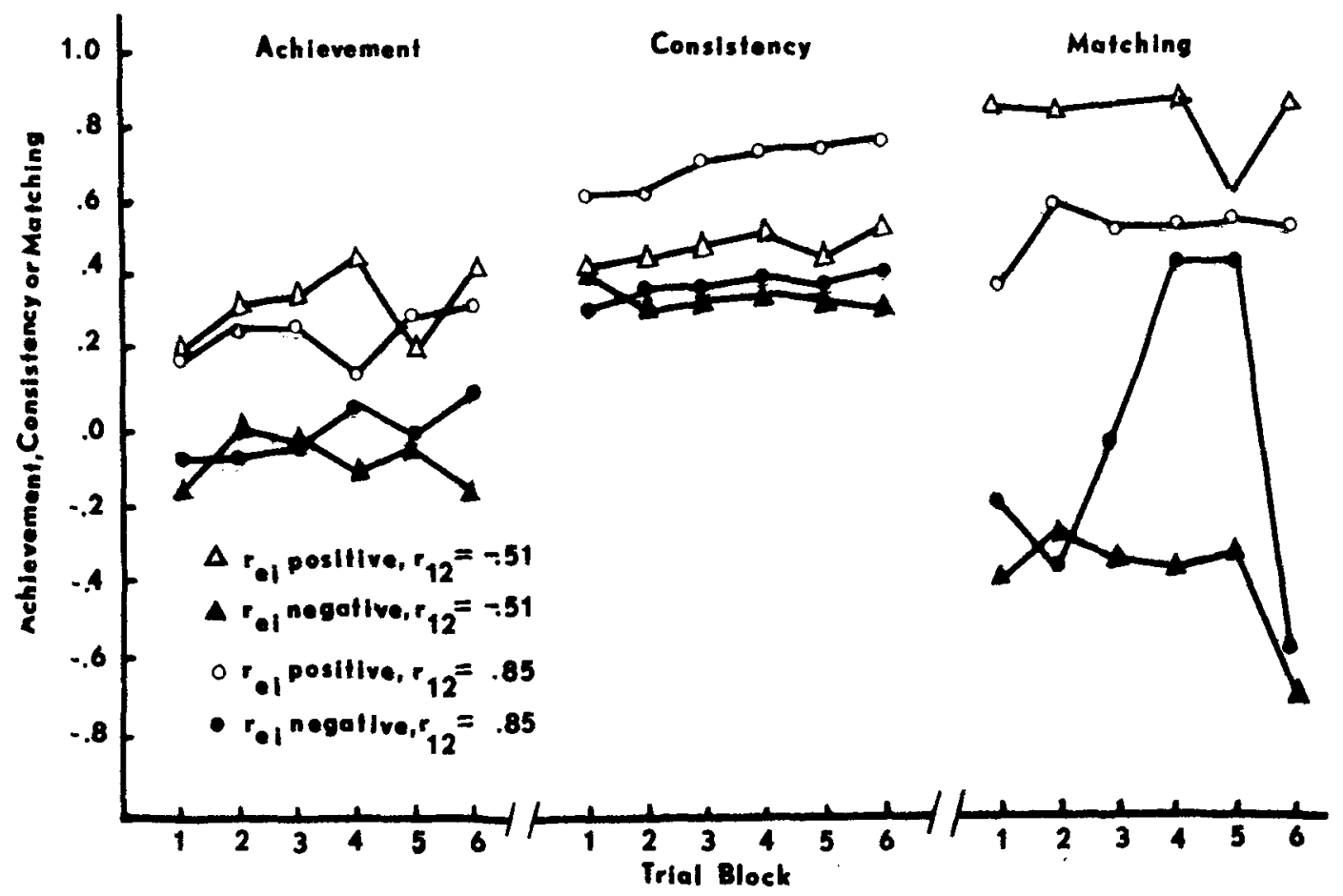

Fig. 8. S achievement, consistency, and matching as a function of the sign of the cue validities, cue intercorrelation, and trial block-Experiment 2.

Analyzing only the dependent variable of achievement, Knowles et al (1971) reported that Ss in the negative redundancy groups learned their task more slowly. There is evidence in the present study that Ss performance in the negative redundancy groups was lower in the first and second trial block (see Figs. 7 and 8). Knowles et al also reported that final achievement in these two groups is quite similar in all conditions; the present study reports similar results, but shows that the reason for the equal achievement in these two groups is quite dissimilar. The present study would indicate that
Ss pay relatively little attention to large differences in beta weights. While the present study cannot be taken as evidence that the only relevant cue for Ss is the cue validities, it does not lend credence to the view that $S s$ are sensitive to beta weights or to the added predictability afforded by the large negative or positive intercorrelation of cues.

The results of the present research are in substantial agreement with Brehmer's transfer of learning experiment (1971) in which the cue-criterion correlations but not the beta weights, or the beta

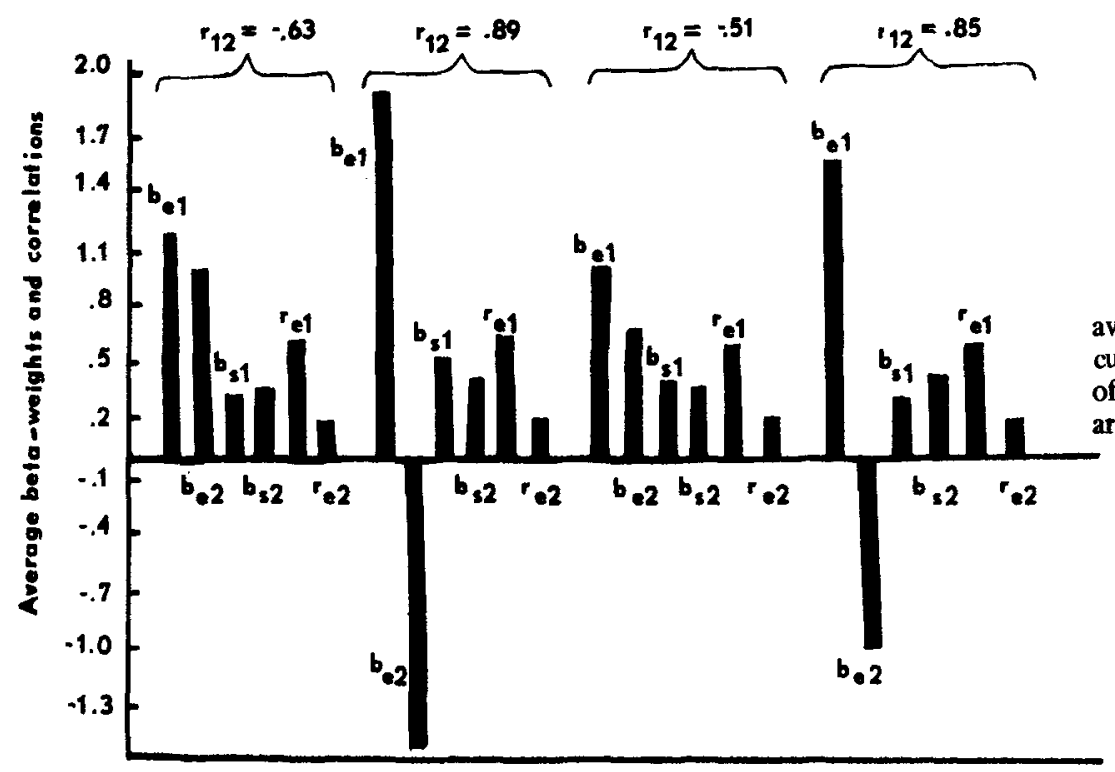

Fig. 9. Cue-criterion beta weights, $b_{e i}$, average cue-judgment beta weights, $b_{s i}$, and cue-criterion correlations, $r_{e i}$, as a function of cue intercorrelation when cue validities are positive. 
weights but not the cue-criterion correlations were changed from the original learning stage to the transfer stage. Performance of Ss (consistency) in Brehmer's study was greater when cue validities were high $\left(\mathrm{r}_{\mathrm{e} 1}=.80, \mathrm{r}_{\mathrm{e} 2}=.40\right)$ than when cue validities were low $\left(\mathrm{r}_{\mathrm{e} 1}=.60, \mathrm{r}_{\mathrm{e} 2}=.00\right)$, regardless of which set of cue validities they were exposed to first. The present Es would agree that Ss' utilization of cues in a MCPL task "cannot be accounted for in terms of the validity of the cues in the task only, regardless of whether the validity is defined in terms of cue-criterion correlations, or in terms of cue-criterion beta weights [Brehmer, 1971, p. 9]." As stated above, the present study does show that performance levels are the same in negative and positive redundancy conditions, but those performance levels are achieved in substantially different ways.

Finally, the results with respect to negative cue validities are in essential agreement with earlier single-cue studies of negative cue validity (e.g., Naylor \& Clark, 1968). As in this previous study utilizing tasks with a single negative cue, $\mathrm{Ss}$ in the present study were totally unable to handle the task situation and the performance indices revealed little or no learning after 150 trials.

\section{REFERENCES}

Armelius, B., \& Lenntoft, K. Effect of cue intercorrelation in a multiple cue probability learning task with different cue validities. Umea Psychological R eports, No, 20, 1970.

Brehmer, B. Cue utilization in multiple cue probability learnir tasks with intercorrelated cues. Umea Psychological Report No. 45,1971

Dudycha, A. L., Dudycha, L. W., \& Schmitt, N. Som overlooked analytical relationships in MCPL. Organization Behavior \& Human Performance, 1974, 11, 222-234.

Dudycha, L., \& Naytor, J, C. Cnaracteristics of the huma inference process in complex choice behavior situations Organizational Behavior \& Human Performance, 1966, 1 110-128.

Knowles, B. A., Hammond, K. R., Stewart, T. R., \& Summers D. A. Positive and negative redundancy in multiple cu. probability tasks. Journal of Experimental Psychology, 1971 90, 157-159.

Naylor, J. C., \& Clark, P. Human inference behavior as function of validity magnitude and sign. Organizationa Behavior \& Human Performance, 1968, 3, 378-399.

Naylor, J. C., \& Schenck, E. A. The influence of cue redundanc on the human inference process for tasks of varying degrees o predictability. Organizational Behavior \& Huma Performance, 1968, 3, 47-61.

Schenck, E. A. A study of the independent effect of cu redundancy upon the human inference process in tasks o varying predictability. Unpublished doctoral dissertation Ohio State University, 1968.

Schenck, E. A., \& Naylor, J. C. Some data concernin performance indices based upon the multiple regression mode when applied in a standard multiple cue situation. Pape present ed at MPA in Chicago, 1965.

Tucker, L. A suggested formulation in the development bs Hursch, Hammond, and Hursch and by Hammond, Hursch and Todd. Psychological Review, 1964, 71, 528-530.
(R eceived for publication January 19, 1974, revision received July 4,1974 .) 